\title{
Arquitecturas de emergencia y continuidad social
}

\section{Emergency architectures and social continuity}

\author{
${ }^{1}$ Alfonso Morone, ${ }^{2}$ Giovanni Verde \\ ${ }^{1}$ DiARC Dipartimento di Architettura Università degli Studi Federico II, Napoli - Italy, \\ alfonso.morone@unina.it \\ 2 DiARC Dipartimento di Architettura Università degli Studi Federico II, Napoli - Italy, \\ gianni.verde@hotmail.it
}

Recepción / Received: 30, 09, 2017

Aceptación / Accepted: 30,10, 2017

Publicado / Published: 26, 12, 2017

Resumen: Los procesos de reconstrucción posteriores a catástrofes deben tender, con la mayor celeridad posible, a proporcionar un primer refugio así como también a iniciar la reconstrucción de la dimensión comunitaria de las áreas destruidas.

Muchas catástrofes han demostrado que una reconstrucción limitada a proveer un simple refugio, puede tener efectos extremadamente negativos en el desarrollo de una comunidad.

Una catástrofe no destruye solo el patrimonio arquitectónico sino que además altera un patrimonio social construido a través de los siglos.

Especialmente las comunidades pequeñas están sujetas, por esto, al riesgo de su completa e irreversible desaparición.

Un planteamiento que no tenga en cuenta, debido a las urgencias, la necesidad de actuar no solo sobre las unidades habitacionales, sino también sobre el patrimonio social, podría afectar dramáticamente al desarrollo de la comunidad afectada.

Si observamos la experiencia italiana, uno de los aspectos más traumáticos posteriores a los últimos sismos, ha sido el rechazo de parte de la población afectada hacia las arquitecturas temporales, containers, etc., en los cuales el concepto de vida comunitaria era inexistente.

Este sentimiento de desapego ha registrado episodios extremos, sobretodo por parte de la población anciana, con sentimientos de confusión ligado a la pérdida repentina de arraigo a la propia comunidad.

Por tanto, la reconstrucción de la comunidad junto con las unidades habitacionales se antoja una necesidad que la estrategia proyectual debe reflejar para dar una respuesta rápida, adecuada y económicamente sostenible.

Este texto desea describir algunas hipótesis desarrolladas en el seno de la investigación universitaria, de soluciones arquitectónicas ante situaciones de emergencia que pretenden, con una acción de transformación social, generar un nuevo tejido comunitario, similar al precedente. Existen algunos factores comunes sobre los cuales se ha desarrollado un proceso proyectual que ha sido aplicado a diversas realidades geográficas, a diferentes contextos territoriales. 
Un primer aspecto es la integración de funciones, de modo que los nuevos módulos habitacionales deben ser, más que elementos autistas, una estructura integrada que estimule las relaciones sociales y que produzca beneficios para sus habitantes.

En una comunidad cada persona desempeña un papel, el cual debe ser recuperado inmediatamente.

Esto significa que los módulos habitacionales deben proveer espacios para oficinas, laboratorios artesanales, negocios, así como también pequeños espacios para huertas que garanticen un autoabastecimiento alimentario.

La dimensión social debe ser acentuada por el modo de agregación de las unidades habitables, por el modo de generar espacios colectivos en donde la comunidad pueda reencontrarse y reconstruirse.

Este planteamiento debe ir acompañado de aquellas estrategias que supongan una reducción de costes, fundamentalmente simplificando los procesos constructivos mediante la autoconstrucción. La autoconstrucción permite un abaratamiento de costes así como la participación de la comunidad local en la regeneración posterior a una catástrofe, lo cual favorece el desarrollo de una nueva cohesión social.

Esto significa que se debe evitar que la población afectada por una catástrofe viva la reconstrucción como una experiencia traumática, y que, asimismo, pueda contribuir a conservar y evolucionar la memoria del patrimonio cultural tangible de la comunidad.

Palabras clave: colaboración; dimensión comunitaria; integración de las funciones; patrimonio social.

\begin{abstract}
The processes of reconstruction after disasters should aim, as quickly as possible, to provide a first refuge as well as to begin the reconstruction of the community dimension of the destroyed areas. Many catastrophes have shown that a reconstruction limited to providing a simple shelter can have extremely negative effects on the development of a community. A catastrophe does not destroy only the architectural heritage but also alters a social heritage built over the centuries. Especially small communities are subject, therefore, to the risk of their complete and irreversible disappearance. An approach that does not take into account, due to the urgencies, the need to act not only about the housing units, but also about the social patrimony, could dramatically affect the development of the affected community. If we look at the Italian experience, one of the most traumatic aspects after the last earthquakes, has been the rejection of part of the affected population towards temporary architectures, containers, etc., in which the concept of community life was non-existent. This feeling of detachment has registered extreme episodes, especially on the part of the elderly population, with feelings of confusion linked to the sudden loss of roots in the community itself. Therefore, the reconstruction of the community together with the housing units seems to be a need that the project strategy must reflect in order to provide a rapid, adequate and economically sustainable response. This text wishes to describe some hypotheses developed within the university research, of architectural solutions in emergency situations that aim, with an action of social transformation, to generate a new community fabric, similar to the previous one. There are some common factors on which a project process has been developed that has been applied to different geographical realities, to different territorial contexts.

A first aspect is the integration of functions, so that the new housing modules should be, more than autistic elements, an integrated structure that stimulates social relations and produces benefits for its inhabitants. In a community each person plays a role, which must be recovered immediately. This means that the housing modules must provide spaces for offices, craft laboratories, businesses, as well as small spaces for vegetable gardens that guarantee food self-sufficiency.
\end{abstract}


The social dimension must be accentuated by the mode of aggregation of the inhabitable units, by the way of generating collective spaces where the community can be reunited and reconstructed. This approach must be accompanied by those strategies that involve a reduction of costs, fundamentally simplifying the construction processes through self-construction. The self-construction allows a reduction in costs as well as the participation of the local community in the regeneration after a catastrophe, which favors the development of a new social cohesion. This means that the population affected by a catastrophe must avoid living the reconstruction as a traumatic experience, and that it can also contribute to preserve and evolve the memory of the tangible cultural heritage of the community.

Keywords: collaboration; community dimension; integration of functions; social heritage.

\section{INTRODUCCIÓN}

El tema de la arquitectura de emergencia está entrelazada estrechamente con la reconstrucción de la dimensión comunitaria de las arquitecturas destruidas.

La simple reconstrucción no parece ser el modo más eficaz para resolver el problema habitacional (Hamdi, 1995)

Muchas catástrofes han demostrado que una reconstrucción limitada a proveer un simple refugio, puede tener efectos extremadamente negativos en el desarrollo de una comunidad.
Una catástrofe no destruye solo el patrimonio arquitectónico sino que además altera un patrimonio social construido a través de los siglos.

\section{La reconstrucción post-emergencia} requiere por esto la participación activa de la población y su implicación directa. (Davis, 2014)

Estas teorías han sido ya ampliamente debatidas en el siglo pasado por arquitectos, urbanistas, sociólogos y teóricos como John Habraken, John F.C. Turner, Nabeel Hamdi, Hassa Fathy y más recientemente reiteradas por Ian Davis. 
Este texto desea describir algunas hipótesis desarrolladas en el seno de la investigación universitaria, de soluciones arquitectónicas ante situaciones de emergencia que pretenden, con una acción de transformación social, generar un nuevo tejido comunitario similar al precedente.

Para esto es necesario escapar de los procesos proyectuales tradicionales. Existe sobretodo la necesidad de mejorar la comunidad directamente en el lugar donde se opera, en estrecha colaboración con ella. (Charlesworth, 2014)

Tenemos, por tanto, la necesidad de aprender, a través de los casos de estudio, nuevos métodos que permitan desarrollar herramientas, y que faciliten, mediante ejemplos mínimos la identificación de nuevas directrices en las que actuar.

Esta voluntad de influir a través de la arquitectura de una manera profunda en los procesos sociales, conforma un nuevo rol de la disciplina en el interior de la cultura contemporánea. (Emmons y Hendrix y Lomholt, 2012)

Los casos de estudios descritos, diferentes según la ubicación geográfica y la naturaleza misma del concepto de emergencia, tienen en común la reflexión sobre la naturaleza del habitar. Se trata de un punto de vista muy introspectivo que desea facilitar soluciones a la dimensión social como factor fundamental en la reconstrucción de la comunidad, partiendo de una idea del alojamiento que acentúa la dimensión comunitaria. El punto de partida es un enfoque extenso al concepto de emergencia habitacional.

Este, en buena parte del mundo, no es un fenómeno temporal, sino una perspectiva estable que tiene que ver con una gran parte de la población (Kennedy, 2005)

Pasando a los aspectos concretos, el punto de partida común en los diferentes casos de estudio es el de la integración de las funciones.

Esto significa que los módulos habitacionales deben proveer espacios para oficinas, laboratorios artesanales, negocios, así como también pequeños espacios para huertas que garanticen un autoabastecimiento alimentario.

La dimensión social debe ser acentuada por el modo de agregación de las unidades habitables, por el modo de generar espacios colectivos en donde la comunidad pueda reencontrarse y reconstruirse. 
Este planteamiento debe ir acompañado de aquellas estrategias que supongan una reducción de costes, fundamentalmente simplificando los procesos constructivos mediante la autoconstrucción.

La autoconstrucción permite un abaratamiento de costes así como la participación de la comunidad local en la regeneración posterior a una catástrofe, lo cual favorece el desarrollo de una nueva cohesión social.

Esto significa que se debe evitar que la población afectada por una catástrofe viva la reconstrucción como una experiencia traumática, y que, asimismo, pueda contribuir a conservar y evolucionar la memoria del patrimonio cultural tangible de la comunidad.

\section{CASOS DE ESTUDIO}

II.a

MÓDULOS HABITACIONALES

PREFABRICADOS COMBINABLES

PARA LA EMERGENCIA

HABITACIONAL EN LOS PAÍSES DEL ÁFRICA SUBSAHARIANA

Las chabolas del área subsahariana son generalmente grandes asentamientos squatter situados cerca de las periferias de las grandes metrópolis.

Estos asentamientos informales tienen su origen en diversos factores, uno de los cuales es el relacionado con el incremento de la población urbana sin un correspondiente aumento de los alojamientos. El África subsahariana representa una de las áreas geográficas, junto con Sudamérica y Asia central, con el mayor número de personas que viven en las citadas chabolas (200 millones, lo que significa que casi un tercio de la población de estas áreas).

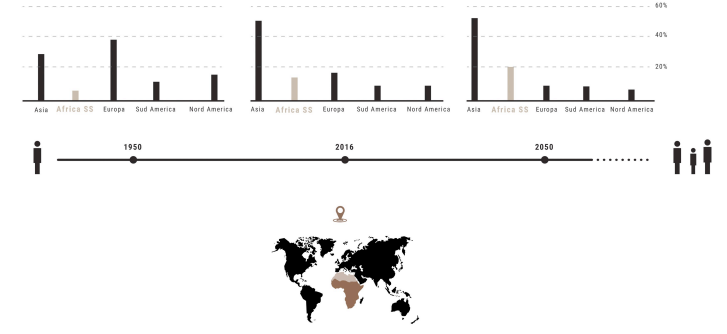

(Tabla 1. Crecimiento de población urbana mundial. Fuente U.N. 2016)

Otro de los factores determinantes de la formación de áreas de chabolas es la migración de las áreas rurales hacia las ciudades en la búsqueda de mejores condiciones económicas. 


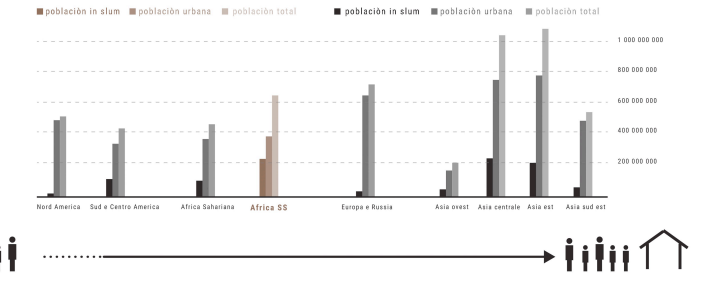

(Tabla 2. Localización población por áreas geográficas. Fuente U.N. 2016)

Precisamente para garantizar la compatibilidad de la intervención con los recursos realmente disponibles, se ha considerado que la intervención debía de ser entendida más como una renovación en vez de una substitución, tomando de esta manera un enfoque que podríamos definir como Upgrade.

A las luces de las consideraciones realizadas, el objetivo ha sido encontrar mecanismos de mediación entre el desastre actual y el estado óptimo, valorables según los standards habiacionales occidentales, utilizando las tecnologías autóctonas involucradas en procesos de industrialización. Se ha intentado extrapolar, desde un escenario con escasez de recursos, aquellos aspectos positivos de ese contexto y que están vinculados al modo de vivir de esa porción de la población; principalmente el aspecto social que representa la casa, el núcleo social y por lo tanto el vacío que representa el verdadero elemento generativo del proceso, junto con la promoción de una economía informal en espacios apropiadamente dimensionados en el interior del núcleo habitacional.

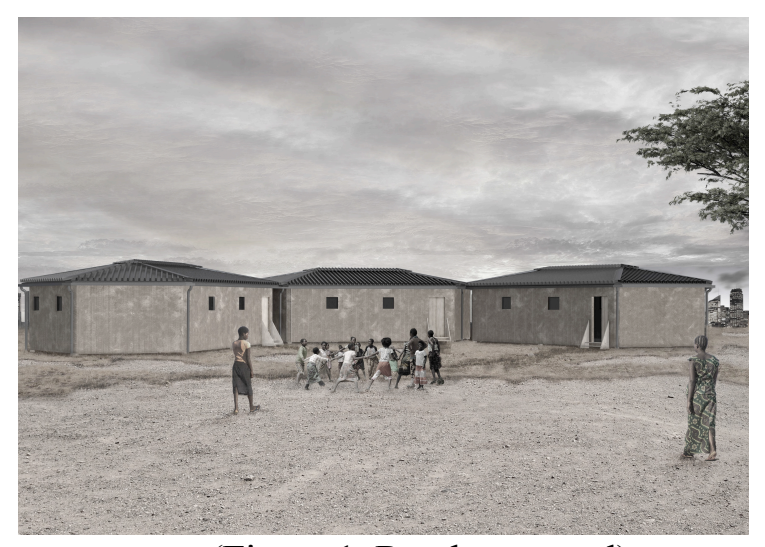

(Figura 1. Render general)

(Noviello, 2017)

La intención era realizar un proyecto progresivo y no revolucionario; introduciendo, dentro de una cultura arquitectónica local consolidada, elementos de innovación tecnológica. El adobe, ampliamente utilizada en la arquitectura rural de este área, es propuesta aquí en forma de paneles modulares prefabricados $\mathrm{y}$ aligerados, contando con una serie de ventajas ligados a la facilidad de montaje, a la reducción de los tiempos de construcción y a la consiguiente economicidad de la propuesta. 


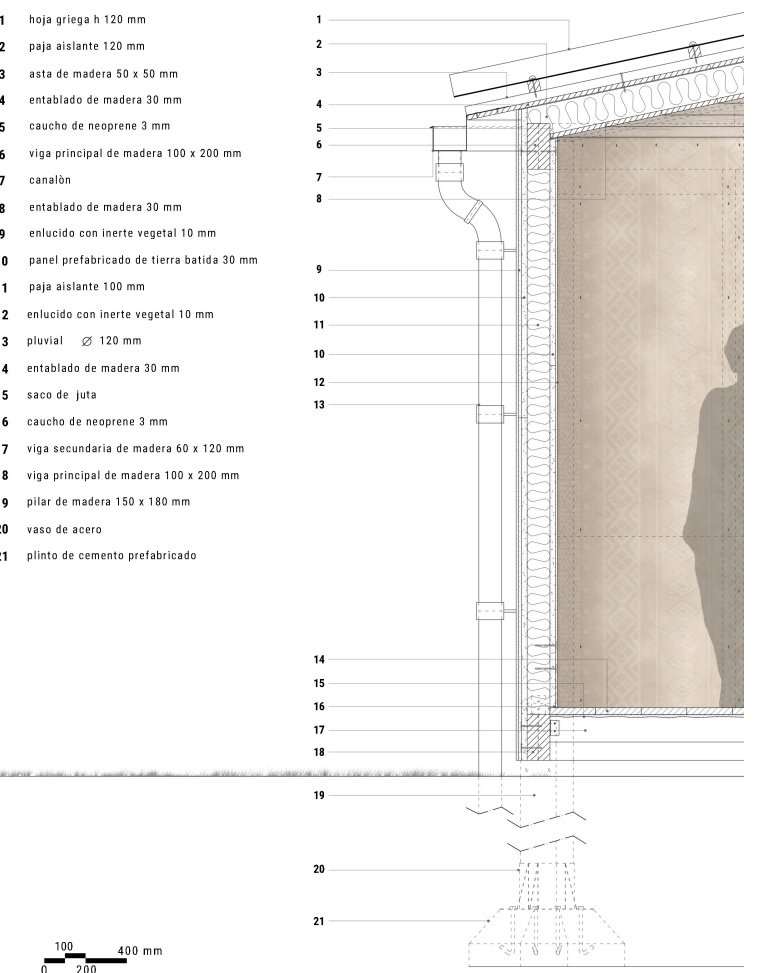

(Figura 2. Dettales / Sección )

(Noviello, 2017)

Del mismo modo, la estructura tradicional de madera ha evolucionado en una estructura del mismo material pero donde intervienen elementos tecnológicamente innovadores representados por las juntas de metal de unión. Por estas razones se puede hablar de proyecto progresivo, como Upgrade, ya que se evoluciona un elemento existente y que deja de esta manera testimonio dentro de la arquitectura rural del área objeto de estudio.

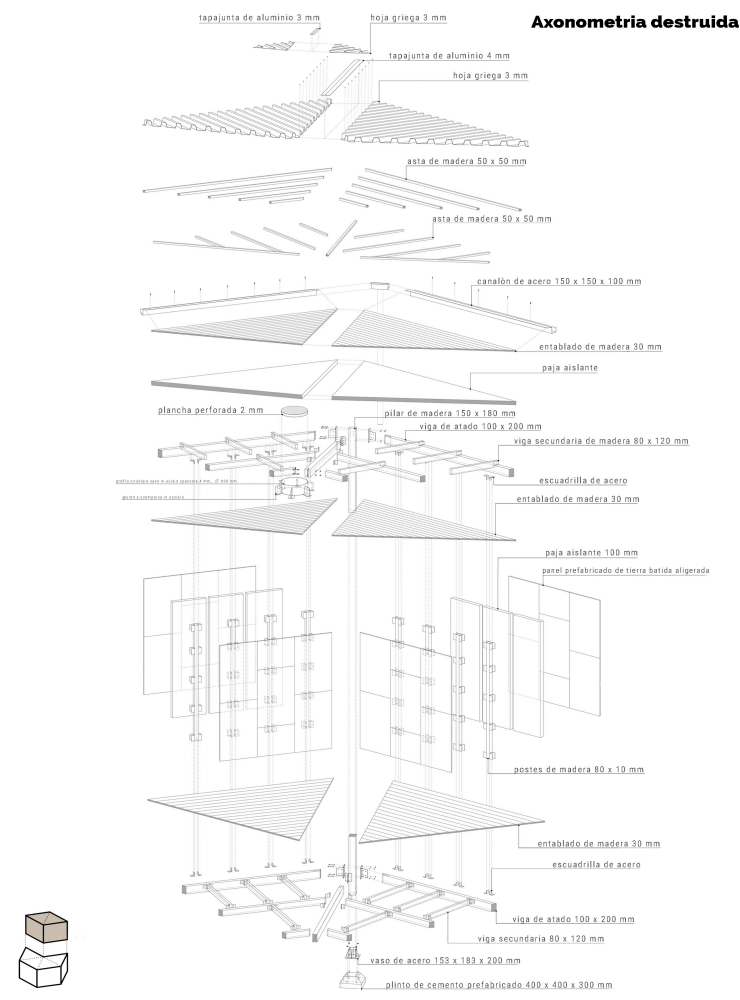

(Figura 3. Axonometria general) (Noviello, 2017)

Se ha planteado la hipótesis de que esta estructura, como ideal de un proceso de prefabricación, pudiese estimular también una localización de una industria local destinada a la realización in situ de los componentes individuales. Por lo tanto, la propuesta tiene la voluntad de favorecer un proceso económico inducido por la solución en si. 


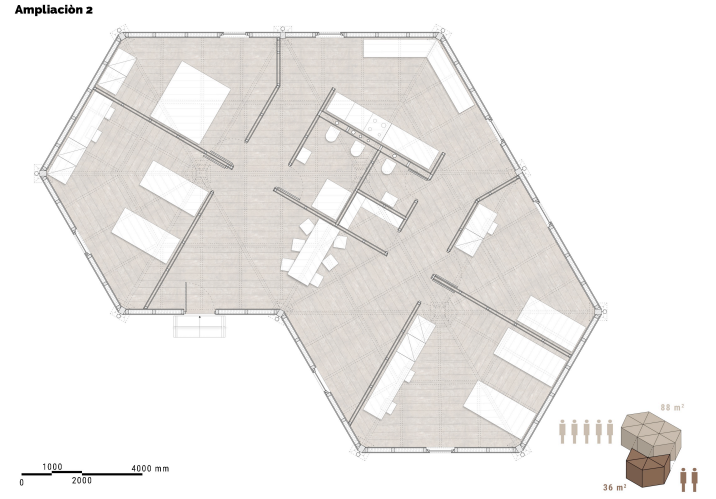

(Figura 4. Plano)

(Noviello, 2017)

\section{II. b}

\section{PROYECTO PARA LA}

\section{RECONSTRUCCIÓN DE POSTGUERRA EN SIRIA}

Historia y cultura han sido elementos fundamentales de este camino, primero cognitivo y luego proyectual. Comprender en su totalidad la arquitectura tradicional, las tipologías edificatorias locales, realizadas por gente sencilla, materiales autóctonos $\mathrm{y}$ técnicas constructivas heredadas durante miles de años, una arquitectura fruto de mezclas étnicas que han definido el área desde la antigüedad.

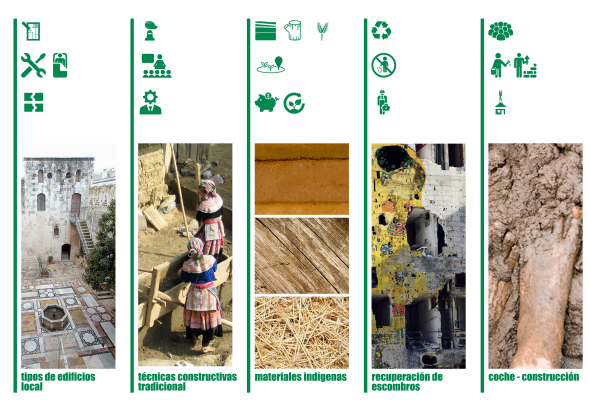

(Figura 5. Tipologías edificatorias locales)

(Bilancone, 2016)

El conocimiento de esta tradición ha permitido la consideración de una nueva tipología de prefabricación, en modelos

evolucionados con respecto a los tradicionales, donde la masa utilizada para las nuevas construcciones pudiese prever el uso de una parte de los materiales derivados de los escombros.

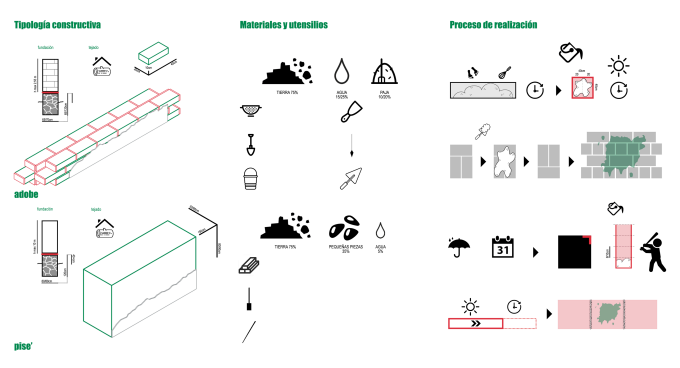

(Figura 6. Tipologia constructiva / Materiales y utensilios / Proceso de realizacion)

(Bilancone, 2016)

Desde un punto de vista social el elemento de relación sobre el que gira la propuesta es el patio. 
El concepto de residencia, de hecho, en la tradición árabe se identifica con la presencia de un patio interno, un espacio de valor introspectivo que ha otorgado a la arquitectura islámica el apelativo de "arquitectura del velo".
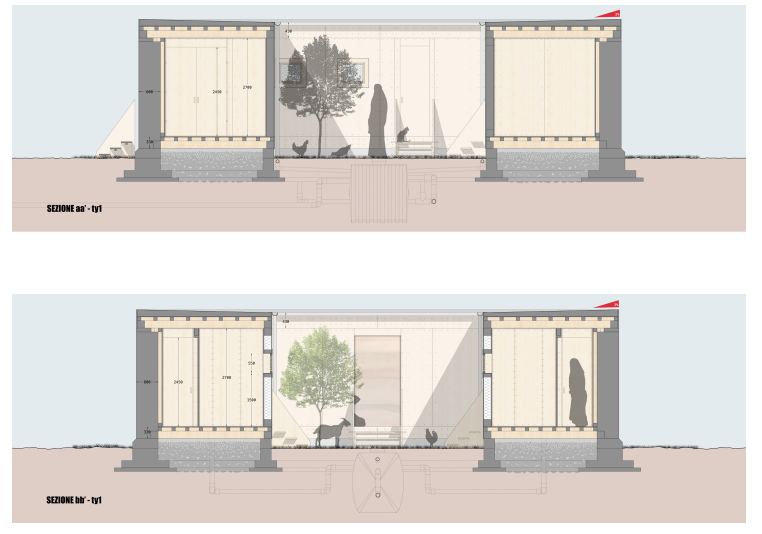

(Figura 7. Sección general ) (Bilancone, 2016)

El patio es un elemento arquitectónico con una larga historia a la cual están asociadas diferentes funciones: los nómadas, principalmente, durante las migraciones en el desierto, colocaban sus cortinas entorno a un espacio central, que brindaba protección al ganado, fuente de sustento económico.

La organización del patio permite la creación de un agradable microclima, sobretodo en las estaciones cálidas y secas, maximizando el sombreado: la presencia de plantas, a menudo árboles frutales, y fuentes en el interior del patio favorece el enfriamiento y la reducción de humedad.
A los patios se les han atribuido un rol cultural y social:

Por un lado, representan un espacio-filtro, destinado a garantizar la privacidad familiar; por el otro, son lugares de encuentro y entretenimiento para los huéspedes y familiares, una suerte de sala de estar al aire libre.

De un estudio realizado sobre la residencia siria, con el fin de establecer una proporción entre el vacío del patio y el lleno de la construcción, se ha establecido una proporción entre el espacio construido y el patio que va de una mínima (1/4), una media $(1 / 3)$ y una máxima $(1 / 2)$.

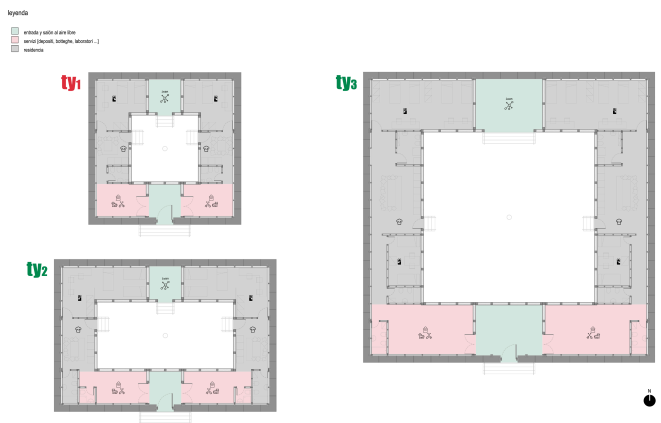

(Figura 8. Planos) (Bilancone, 2016)

Los materiales previstos para la realización de las unidades habitacionales son materiales autóctonos y locales, así como también las técnicas constructivas utilizadas. 
Para la realización del muro perimetral externo se ha utilizado el adobe: esta técnica, de hecho, tradicionalmente ha sido utilizada para realizar muros, revestir fachadas y entre ladrillos como mortero.

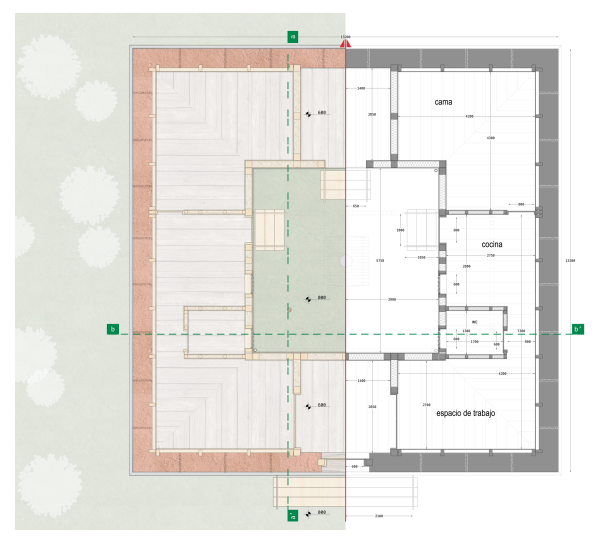

(Figura 9. Plano)

(Bilancone, 2016)

Las técnicas constructivas usadas para la ejecución de los muros perimetrales fueron esencialmente dos: el adobe y el tapial. Con la primera técnica es posible producir ladrillos, con una mezcla de tierra, paja y agua; esta masa se vierte en un molde, dividido en el medio, del cual es posible obtener dos ladrillos; el molde se debe dejar reposar entre 5/10 días, hasta que se complete el secado.

El tapial, mezcla de tierra, grava y agua, es utilizado para la realización de bloques mediante un encofrado de madera; dentro del encofrado se debe compactar la tierra en capas de 8/10 cm; piedras pequeñas, colocadas entre un tapial y otro, garantizan la conglomeración de la masa. Esta masa se puede realizar mediante la utilización de materiales provenientes de escombros resultados de sucesos bélicos.

El muro perimetral está compuesto de adobe estabilizado, compuesta la masa por: el $71 \%$ de tierra, el $31 \%$ de grava, $1,8 \%$ de granulado de hormigón reciclado (proveniente de escombros) y el 5\% de agua; la técnica constructiva utilizada es la propia del tapial; al final del tabique, ya insertado en un encofrado, un montante vertical de abeto, sobre el cual se clava un panel, también de abeto, de cara a la protección del muro en contacto con el terreno o en contra del paso de vegetación.

\section{II.c}

\section{SOLUCIÓN A LA EMERGENCIA}

HABITACIONAL DE LOS

\section{BARRACONES DE DHARAVI}

Dharavi es un barrio pobre ubicado en la ciudad de Mumbai que se desarrolla en un área de aproximadamente $1.7 \mathrm{~km}^{2}$. Hoy en día es uno de los barracones más grandes del mundo y el más grande de Asia, con una población estimada entre 600.000 y un millón de habitantes. 
El espacio es el verdadero problema del barrio, sobretodo en cuanto a su organización.

La posibilidad de componer el mismo espacio habitable mediante el ensamblaje de unidades combinables es seguramente una manera elemental pero eficaz de organizar los espacios disponibles y tenerlos bajo control.

La principal modalidad de construcción en los barracones es la autoconstrucción. Obviamente las razones son de tipo económico, pero no se debe subestimar el aspecto cultural:

Dharavi es una realidad que funciona y en la que los habitantes hacen por producir, vender, vivir.

La característica de esta sociedad es precisamente el intercambio y el compromiso entre generaciones. Un proyecto de autoconstrucción de barrios debe poder contar con la cooperación de la comunidad para alcanzar un objetivo común, que no es otra cosa sino mejorar la vida del lugar.

Las imágenes de Dharavi subrayan la total falta de planificación del barrio.

El proceso proyectual nace a partir de un análisis socio-económico en el cual se muestra evidente el carácter de una comunidad muy unida, colaborativa, fundada sobre la cooperación y el intercambio.

La estructura está proyectada pensando en las técnicas constructivas más intuitivas $\mathrm{y}$ tradicionales, en vista de la fase de autoconstrucción

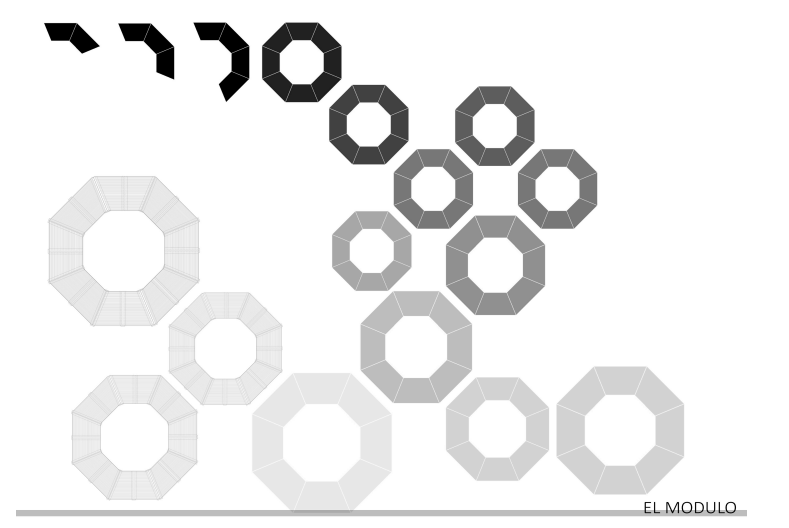

(Figura 10. Modulo)

(Pinto, 2014)

Se trata de una estructura de madera con armazones simples.

El material de construcción elegido es la madera de Pyinma, una especie autóctona, de fácil disponibilidad local, adaptada a la construcción, resistente al ataque de insectos, de crecimiento rápido, muy económica.

La estructura es formada por cinco pilastras, ancladas a la cimentación a través de placas atornilladas internas, y cinco vigas de base.

La cubierta consiste en tres armazones de altura ascendente, conectados entre sí mediante una viga. 


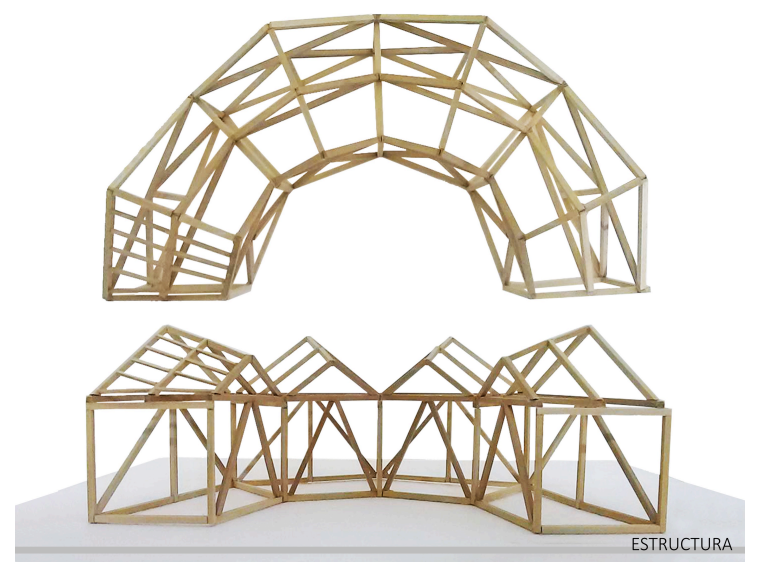

(Figura 11. La estructura)

(Pinto, 2014)

Todos las conexiones son de tipo mecánico, previendo por esto placas atornilladas para el anclaje.

Para garantizar una mayor estabilidad estructural los módulos se pueden agregar en un máximo de cuatro, después la estructura se duplica.

El módulo individual tiene una superficie transitable de aproximadamente $19 \mathrm{~m}^{2}$.

El módulo puede ser cerrado e independiente como también puede conectarse a los otros eliminando cerramientos.

Gracias a la altura variable de los armazones es también posible aumentar la superficie: aprovechando las cadenas de los dos armazones más altos y la altura habitable del bajocubierta, es posible crear con un simple entablado un generoso altillo cercano a los 9 $\mathrm{m}^{2}$ de superficie.
Entre los armazones hay viguetas de unión que, más allá de la función estática, sirven de apoyo para el aislamiento térmico vinculado a la chapa metálica.

La cubierta está realizada en chapa zincada ondulada: un material ya conocido, de fácil puesta en obra, económico, impermeable, útil en la recogida del agua de lluvia.

El aislamiento térmico está previsto en papel reciclado contenido en sacos de yute, solución muy económica, facilmente reproducible y ecosostenible utilizando la propiedad aislante de un material muy difundido y a menudo

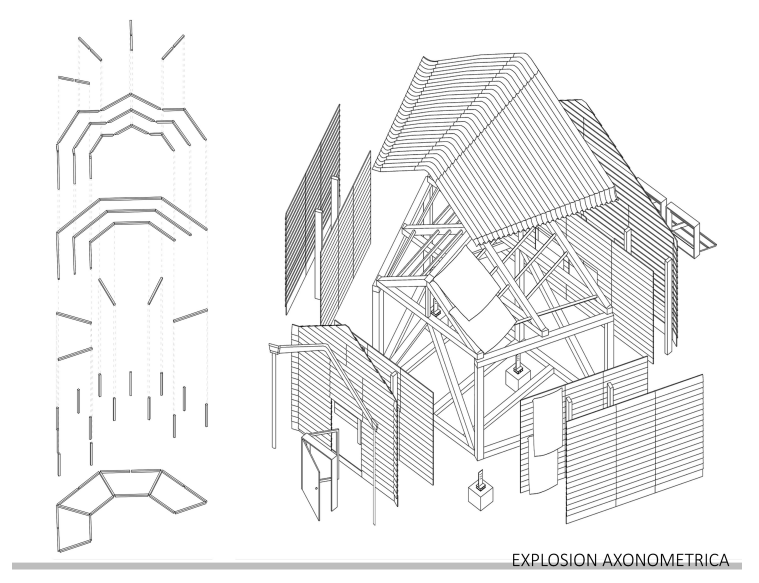

(Figura 12. Axonometria) (Pinto, 2014)

El revestimiento externo está previsto en Clapboards: listones largos y delgados de madera, posados de manera que se solape una parte del lado largo - técnica similar a a la colocación de las tejas - para tener una mayor protección contra el agua y los agentes atmosféricos. 


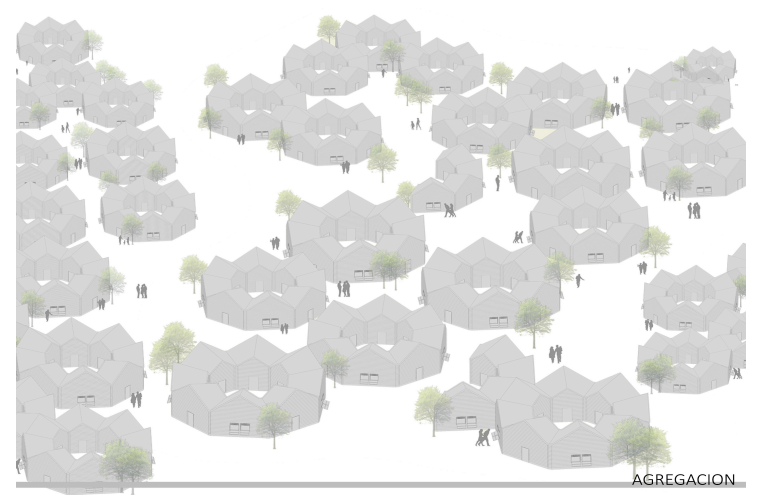

(Figura 13. Modelo de ensamblaje de unidades combinables)

(Pinto, 2014)

El entablado interno permite rematar el intradós de la estructura y crea una capa intermedia donde pueden ser alojados otros sacos aislantes.

La canaleta para la recogida de agua de lluvia sigue la geometría de la falda e impide a a la lluvia correr a lo largo de la fachada.

La versatilidad del proyecto se orienta tanto al aspecto funcional del espacio así como también al aspecto tecnológico plegándose a las exigencias, a las posibilidades económicas y a los hábitos de sus ocupantes.

\section{II.d}

\section{SISTEMA PREFABRICADO MODULAR EN FIBRA NATURAL}

El proceso proyectual del sistema prefabricado modular en fibra natural nace de la exigencia de alcanzar determinados objetivos como: prefabricación, capacidad de agregación, expansión y modulación.

Además, el proyecto del sistema habitacional es temporal y dedicado a zonas en vías de desarollo o zonas de emergencia; eso implica la utilización de materiales naturales totalmente reciclables: el bambú. El proceso de diseño se inicia con la individualización de un módulo standard que define los espacios mínimos de alojamiento dedicados a cada persona.

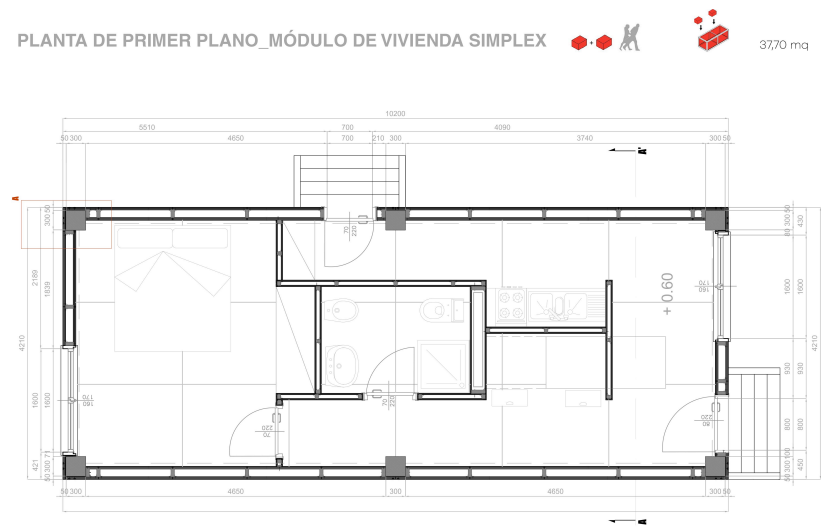

(Figura 14. Plano tipo)

(Bagnato, 2016)

Un módulo es parte interna de una estructura combinable y expandible. Este sistema puede seguir creciendo incluso después de su propia construcción. La elección del material de construcción recae sobreel bambú porque es un material altamente ecológico y reciclable. Crece rapidamente, acumula un alto nivel de co2, tiene excelentes propiedades mecánicas, ligereza y facilidad para trabajarlo. 


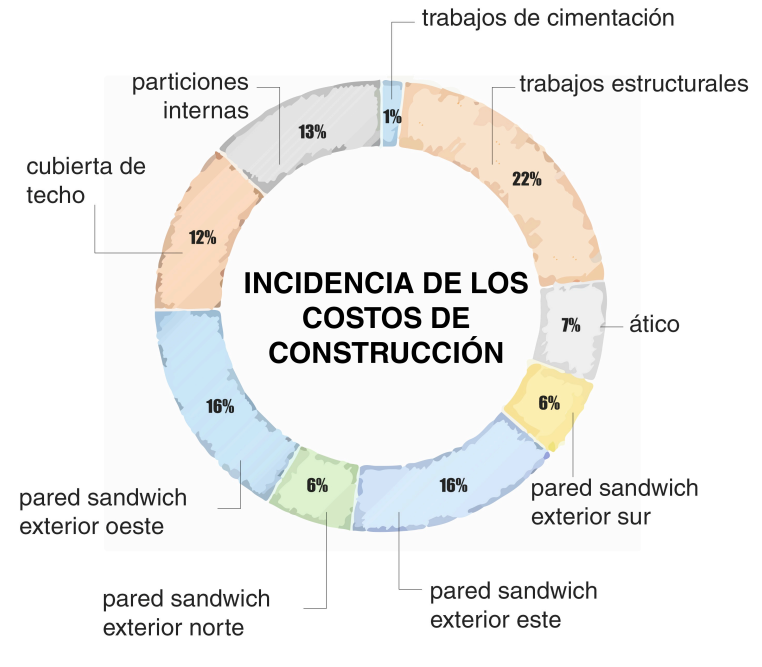

(Tabla 3. Incidencia de los costos de construcción)

(Bagnato, 2016)

El módulo standard es de $37.70 \mathrm{~m}^{2}$ dedicado a dos usuarios donde los cimientos están realizados sobre plintos a encofrado perdido en chapa ondulada; la cara superior del plinto está vinculada a la estructura de bambú mediante planchas plegadas de unión.

La estructura es cajeada y constuída de pilastras, vigas principales y secundarias en bambú laminado utilizando como elementos de unión placas plegadas de acero.

El suelo está compuesto por vigas, aislamiento de vidrio celular y un entablado de bambú compuesto de tres estratos cruzados de $30 \mathrm{~mm}$ de espesor.

Los cerramientos exteriores están compuestos de paneles de bambú laminado en 5 estratos cruzados en horizontal y vertical de $40 \mathrm{~mm}$ de espesor, unidos tras ellos por montantes igualmente de bambú laminado.

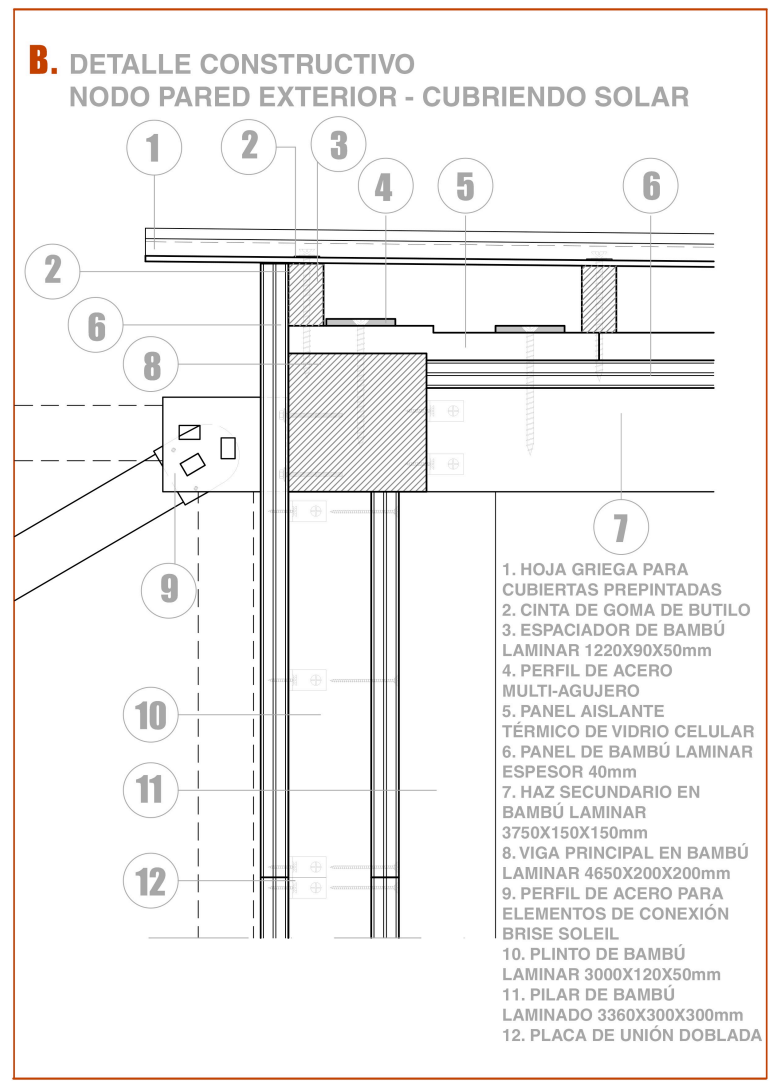

(Figura 15. Dettales / Sección)

(Bagnato, 2016)

Como aislante están previstos materiales de deshecho situados entre los dos paneles.El forjado inferior está compuesto de paneles de bambú laminado en 5 estratos de $40 \mathrm{~mm}$ de espesor, un estrato termoaislante en vidrio celular, perfiles que protegen el panel aislante, separadores de bambú laminado y chapa grecada.

Las particiones internas tienen el mismo procedimiento constructivo que las externas; están constituidas de paneles que utilizan 
como elemento de unión un montante de bambú laminado.

La planta interna se articula situando el núcleo de servicios en el centro del módulo y estableciendo también el diseño de algunos elementos de mobiliario como la mesa de trabajo móvil.

Las paredes sandwich del este y oeste cuentan con elementos brise soleil que desempeñan diversas funciones dependiendo de su ubicación. Su posición horizontal actúa como elemento de unión con otros sistemas habitables, mientras que la oblicua delimita una zona en el terreno que puede utilizarse para el cultivo privado.

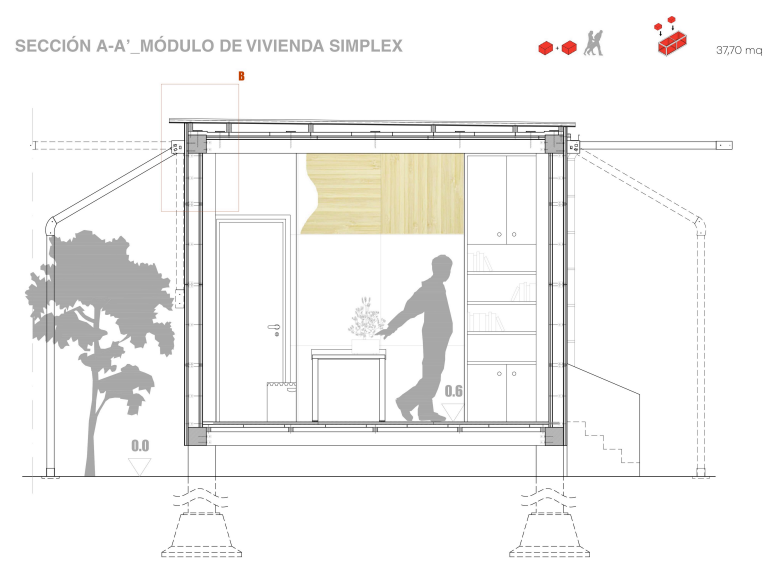

(Figura 16. Sección general)

(Bagnato, 2016)

Mediante la combinación de dos estructuras los sistemas habitables se expanden pudiendo acoger en su interior grupos de familias más numerosas.
De igual manera a los sistemas habitables están previstos sistemas aditivos para laboratorios de modo que con la agregación se puedan crear auténticos y propios barrios con una economía autónoma.

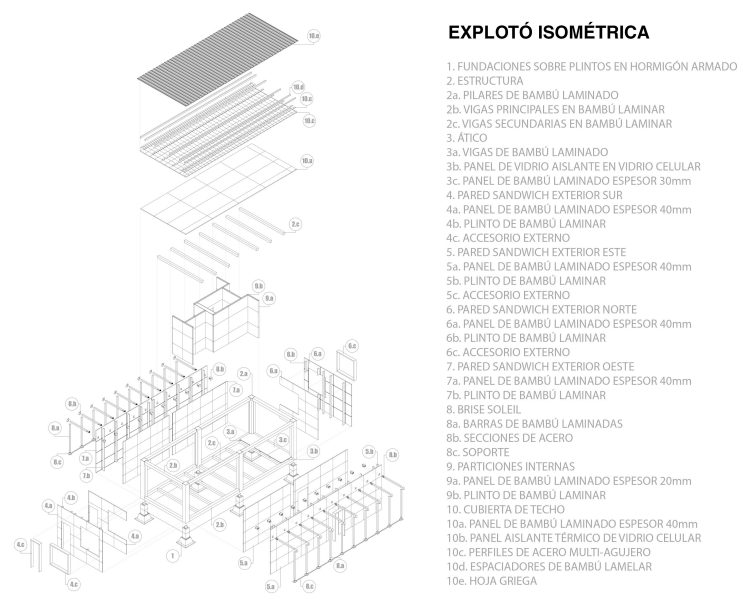

(Figura 17. Vista isometrica)

(Bagnato, 2016)

\section{II.e \\ DISEÑO DE EMERGENCIA EN \\ BAMBÚ Y BALSA PARA SUDAMÉRICA}

La estructura principal es una pirámide invertida, con el vértice como único punto de cimentación, la rotación de la estructura viene limitada por la escalera o pasarela. La cimentación centrada en un punto abarata los costes y permite la utilización agrícola de la superficie libre envuelta por la construcción. El proyecto presenta una mezcla de técnicas locales y modernas. El módulo habitacional, 
pensado para 4 personas, está constituido por módulos de 4x4 m combinados. La forma implica una mayor superficie a la cubierta que garantiza refugio ante los agentes atmosféricos y una superficie menor en la parte inferior incluyendo la posibilidad de almacenamiento aprovechando las paredes inclinadas.

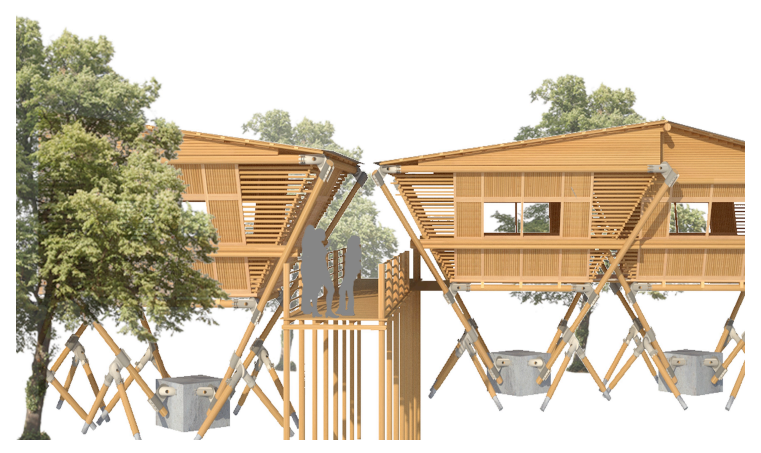

(Figura 18. Render general)

(Parlato, 2013)

El elemento estructural principal es el nudo de acero inoxidable compuesto por 3 placas gruesas de $10 \mathrm{~mm}$, la central estás soldada con vidrio que deja intacta la vara de bambú que desde el vértice llega hasta el plano de cubierta sin ninguna interrupción.

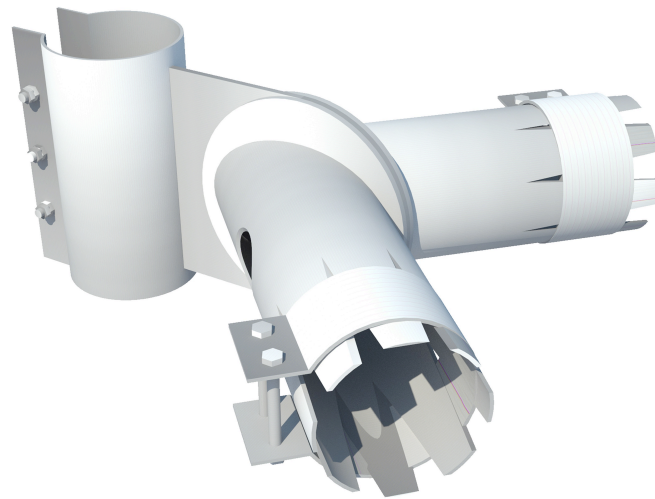

(Figura 19. Elemento estructural de acero inoxidable)

(Parlato 2013)

A las placas externas se les suelda, a una de ellas un tubular hueco de $200 \mathrm{~mm}$ de diámetro seccionado a $45^{\circ}$ para permitir la soldadura, y a la otra un tubular a $90^{\circ}$ con dientes para permitir, una vez insertado el bambú, apretar el tubular a través de un collar hasta la cabeza del bambú rigidizado mediante hormigón. Se unen entre sí por medio de una brida para garantizar el alineamiento y además, existe una ranura que permite introducir el bulón, y mediante herramientas especiales fijar los 3 elementos y amarrar el conjunto una vez determinada la inclinación. En el nudo convergen 3 varas de bambú, dos fijadas a $90^{\circ}$ mientras que la tercera, proveniente del vértice, es regulable. 


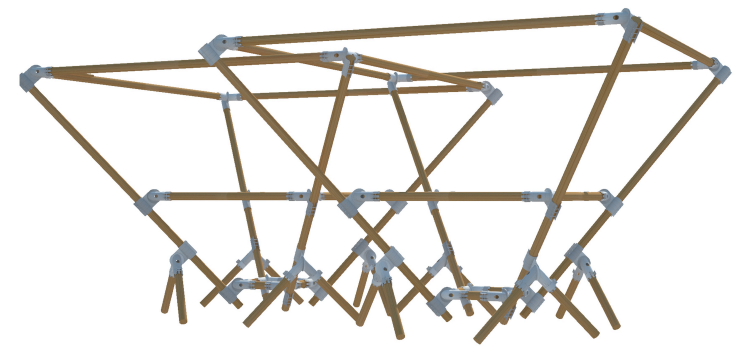

(Figura 20 La estructura principal es una pirámide invertida)

(Parlato, 2013)

El nodo intermedio, entre el pavimento y la cimentación, gira $180^{\circ}$ y porta las varas armadas hasta el suelo, dando una mayor estabilidad al prisma, mientras la vara de la cimentación está inmersa en el plinto sobresaliente. En lo concerniente a las conexiones internas estas son realizadas con técnicas tradicionales con la particularidad de estar desconectadas entre ellas para poder efectuar manutenciones y substituciones.

\section{CONCLUSIONES Y RECOMENDACIONES}

Una aproximación a las emergencias habitativas, que no tenga en cuenta la necesidad de actuar no solo sobre el patrimonio arquitectónico, sino también sobre el patrimonio social, podría tener efectos catastróficos sobre el futuro de la comunidad.

Sobretodo las comunidades más pequeñas son susceptibles, por esto, de desaparecer total e irreversiblemente.

La reconstrucción de la comunidad junto con los elementos habitacionales, se vuelve una necesidad que la estrategia proyectual debe cuidadosamente reflejar con el objetivo de dar una respuesta rápida, adecuada $\mathrm{y}$ sostenible en términos económicos.

Este proceso necesita de ejemplos que alumbren una nueva modalidad proyectual, en donde las exigencias sociales estén en el centro de la estrategia proyectual.

Un enfoque a las emergencias de vivienda, que no toma en cuenta la necesidad de actuar además de los activos inmobiliarios de vivienda, incluidos los activos sociales, podría tener efectos catastróficos en el futuro de las comunidades locales.

Las comunidades especialmente más pequeñas están sujetas al riesgo de su desaparición total e irreversible.

La reconstrucción de la comunidad junto con la vivienda es, por lo tanto, una necesidad que la cultura de planificación debe reflejar cuidadosamente a fin de proporcionar respuestas rápidas, adecuadas y 
sostenibles en términos económicos.

Este proceso necesita ejemplos que indiquen un nuevo modo de diseño, donde las necesidades sociales están en el corazón del enfoque de diseño.

De acuerdo con la bibliografía de referencia utilizada, se cree que la atención de la investigación de diseño debe enfocarse en el desarrollo de un proceso de diseño apropiado. Precisamente por esta razón, los ejemplos utilizados representan un claro ejemplo de cómo los resultados formales y los aspectos tecnológicos deben guiarse por elecciones locales, mientras que el objetivo metodológico sigue siendo único: el de fomentar, a través del proyecto del hogar, la regeneración de un Contexto de la comunidad.

\section{AGRADECIMIENTOS}

Capitulo II.a

Salvatore Noviello

MÓDULOS

HABITACIONALES

PREFABRICADOS

COMBINABLES

PARA

LA

EMERGENCIA

HABITACIONAL EN LOS PAÍSES DEL

ÁFRICA SUBSAHARIANA

Master Thesis in Architecture, Supervisor prof. Alfonso Morone, DiARC Department of Architecture, Università degli Studi di Napoli Federico II

Tabla 1, Tabla 2, Figura 1, Figura2, Figura 3, Figura 4.

Capitulo II. b

Loredana Bilancione,

PROYECTO PARA LA
RECONSTRUCCIÓN DE POSTGUERRA

EN SIRIA

Master Thesis in Architecture, Supervisor

prof. Alfonso Morone, DiARC Department of Architecture, Università degli Studi di Napoli

Federico II

Capitulo II.cFabiana Pinto

SOLUCIÓN A LA EMERGENCIA HABITACIONAL DE LOS BARRACONES DE DHARAVI

Master Thesis in Architecture, Supervisor prof. Alfonso Morone, DiARC Department of Architecture, Università degli Studi di Napoli Federico II

Figura 5, Figura 6, Figura 7, Figura 8, Figura 9, Figura 18 , Figura 19, Figura 20.

Capitulo II.c

Fabiana Pinto

SOLUCIÓN A LA EMERGENCIA HABITACIONAL DE LOS BARRACONES DE DHARAVI

Master Thesis in Architecture, Supervisor prof. Alfonso Morone, DiARC Department of Architecture, Università degli Studi di Napoli FedericoII.

Figura 10, Figura 11, Figura 12, Figura 13

Capitulo II.d

Flavia Bagnato

SISTEMA PREFABRICADO MODULAR EN FIBRA NATURAL

Master Thesis in Architecture, Supervisor prof. Alfonso Morone, DiARC Department 
of Architecture, Università degli Studi di Napoli Federico II

Figura 14, Tabla 3, Figura 15, Figura 16 ,

Figura 17

Capitulo II.e

Angela Covone

DISEÑO DE EMERGENCIA EN BAMBÚ

Y BALSA PARA SUDAMÉRICA

Bachelor in Architecture, Supervisor prof. Alfonso Morone, DiARC Department of Architecture, Università degli Studi di Napoli Federico II

\section{REFERENCIAS}

Charlesworth,

E.

(2014).

Humanitarian Architecture: 15 stories of architects working after natural disasters. Abingdon,UK: Routledge.

Davis,I.(2013,OctoberDecember). Architects and Emergencies Boundaries International Architecture Magazine, 10 (III), 67-74.

van Der Plas, E. Frerks, G. Goldewijk, B.K. (2011). Cultural Emergency in Conflit and Disaster. Rotterdam, Nederland: NAi Publisher.

Emmons, P. Hendrix, J. Lomholt, J.(2012). The Cultural Role of Architecture. Contemporary and Historical Perpestctive. Abingdon,UK: Routledge.

Fathy, H. (2000). Architecture for the Poor: An Experiment in Rural Egypt. Chicago, Illinois: University Of Chicago Press.

Habraken, N. J. (1999). Supports: An
Alternative to Mass Housing. Gateshead, UK: Urban International Press.

Hamdi, N. (1995). Housing Without Houses. Partecipation, Flexibility, Enablement. London, UK: Intermediate Techonology Publication.

Khalili, N. Outram, I. (2008). Shelter and Eco-Village: Manual -How to Build Your Own with Superadobe Emergency Sandbag / Earthbags. Hesperia, California: Cal Earth Press.

Kennedy,J. (2005).Challenging Camp Design Guidelines Forced Migration Review. 23, 46-47.

Rosa, M. Weiland, U. (2013).Handmade Urbanism: Mumbai, São Paulo, Istanbul, Mexico City, Cape Town. From Community Initiatives to Participatory Models. Berlin, Germany: Jovis publisher.

Turner, J.F.C. (1976). Housing By People: Towards Autonomy in Building Environments. London, UK: Marion Boyars.

Turner, J.F.C.(1972). Freedom to Build : Dweller Control of the Housing Process. Basingstoke, UK: The Macmillan Company. 